\title{
Determinação das fontes de repasto sanguíneo de Culicoides Latreille (Diptera, Ceratopogonidae) em áreas rurais do norte do estado do Maranhão, Brasil
}

\author{
Gaudino Marco Cantanhede Gusmão ${ }^{1}$ \\ Elias Seixas Lorosa ${ }^{2}$ \\ Gustavo Almeida Brito ${ }^{3}$ \\ Leandro Santos Moraes ${ }^{3}$ \\ Vagner de Jesus Carneiro Bastos ${ }^{3}$ \\ José Manuel Macário Rebelo ${ }^{3 *}$ \\ ${ }^{1}$ Programa de Pós-Graduação, Mestrado em Biodiversidade e Conservação \\ Universidade Federal do Maranhão, São Luís - MA, Brasil \\ ${ }^{2}$ Laboratório Nacional e Internacional de Referência em Taxonomia de Triatomíneos \\ Instituto Oswaldo Cruz, Fundação Oswaldo Cruz, Rio de Janeiro - RJ, Brasil \\ ${ }^{3}$ Laboratório de Entomologia de Vetores, Universidade Federal do Maranhão \\ Departamento de Patologia, Praça Madre Deus, 02, CEP 65025-560, São Luís - MA, Brasil \\ * Autor para correspondência \\ macariorebelo@uol.com.br
}

Submetido em 11/03/2014

Aceito para publicação em 15/12/2014

\section{Resumo}

O conteúdo estomacal de fêmeas do gênero Culicoides foi estudado para determinar suas fontes alimentares sanguíneas e o grau de relações estabelecidas entre esses insetos e seus hospedeiros. Os espécimes foram capturados em armadilhas luminosas tipo CDC no período entre março de 2009 e fevereiro de 2010, em duas localidades rurais da ilha de São Luís-MA. Foram capturadas 930 fêmeas ingurgitadas, pertencentes a 12 espécies de Culicoides. Foi utilizado o exame da reação da precipitina, que revelou o sangue de sete tipos diferentes de vertebrados que haviam sido sugados, sendo os mais frequentes: ave $(41,9 \%)$, roedor $(21,2 \%)$, cão $(15,4 \%)$ e ser humano (7,3\%). Nas reações duplas predominaram ave/cão $(20 \%)$ e cão/gato, cão/gambá, ave/roedor, gambá/roedor e gato/humano (13,3\% cada). Dentre as espécies de Culicoides encontradas, C. paucienfuscatus Barbosa, 1947 destacou-se, por ter sugado apenas sangue de aves. As 11 espécies restantes sugaram sangue tanto de animais domésticos como de sinantrópicos, sendo que, destas, quatro também sugaram o sangue humano. Os resultados permitiram concluir que $C$. paucienfuscatus apresentou relações específicas, foi considerada ornitófila e as outras espécies foram generalistas. A presença desses animais em ambiente peridoméstico é um fator que favorece a manutenção dos Culicoides nos povoados rurais e a presença do ser humano como um dos vertebrados mais sugados indica que além de fazer parte da dieta das fêmeas de Culicoides, pode participar de eventuais ciclos epidemiológicos de parasitas, devido ao hábito eclético desses insetos.

Palavras-chave: Hematofagia; Hospedeiros; Maruins; Vetor 


\section{Abstract}

Determination of blood meal sources of Culicoides Latreille (Diptera, Ceratopogonidae) in rural areas of the northern Maranhão state, Brazil. The stomach contents of females from the genus Culicoides were studied to determine their blood food sources and the degree of relations established between these insects and their hosts. The specimens were captured by using CDC light traps within the period from March 2009 to February 2010, in two rural towns in the island of São Luís, Maranhão, Brazil. A total of 930 engorged females were captured, belonging to 12 Culicoides species. Precipitin reaction examination was used, which revealed the blood from 7 different vertebrate types that had been sucked, and the most frequent were: bird (41.9\%), rodent $(21.2 \%), \operatorname{dog}(15.4 \%)$, and human being $(7.3 \%)$. In double reactions there was a predominance of bird/ $\operatorname{dog}(20 \%)$ and $\operatorname{dog} /$ cat, dog/opossum, bird/rodent, opossum/rodent, and cat/human being (13.3\% each). Among the Culicoides species found, C. paucienfuscatus Barbosa, 1947 stood out, because it only sucked blood from birds. The 11 remaining species have sucked blood both from domestic and synanthropic animals, and 4 out of them also sucked human blood. The results allowed us to conclude that $C$. paucienfuscatus showed specific relations, it was considered as ornithophilous and the other species were generalist. The presence of these animals in a peridomestic environment is a factor that favors the maintenance of Culicoides in rural villages and the presence of human being among the most sucked vertebrates indicates that besides being included in the diet of female Culicoides, it may participate in occasional epidemiological cycles of parasites, due to the eclectic habit of these insects.

Key words: Biting midges; Hematophagy; Hosts; Vector

\section{Introdução}

O gênero Culicoides Latreille constitui um grande grupo de dípteros nematóceros da família Ceratopogonidae, compreendendo 1.387 espécies distribuídas pelo mundo, sendo 1.343 existentes e 44 fósseis (BORKENT, 2014). Destas, pelo menos 266 ocorrem na região Neotropical (BORKENT; SPINELLI, 2007) e mais de 100 espécies distribuem-se no Brasil (WIRTH; BLANTON, 1973; WIRTH et al., 1988; FELIPPE-BAUER; OLIVEIRA, 2001; APARÍCIO et al., 2011).

As espécies deste gênero estão entre os menores dípteros do mundo, com tamanho medindo de um a quatro milímetros de comprimento. Possuem o tórax curvado sobre a cabeça, e na maioria das espécies, as asas apresentam um padrão de manchas cinzentas e brancas que permite identificá-las taxonomicamente. Entretanto, para algumas espécies é necessário recorrer a análises morfológicas mais complexas (MEISWINKEL et al., 2004).

As fêmeas são hematófagas e muitas espécies são essencialmente zoofílicas, obtendo sangue de mamíferos, aves, répteis e anfíbios (MELLOR et al., 2000) para maturação dos ovários. Outras espécies são antropofílicas e podem causar problemas à saúde humana (BORKENT; SPINELLI, 2007).

O estudo do conteúdo estomacal é importante por possibilitar a identificação de animais que funcionam efetivamente como fonte de alimento sanguíneo aos Culicoides e, o entendimento sobre o grau de preferência que determinadas espécies possam desenvolver por seus respectivos hospedeiros (MISSAWA et al., 2008). Essas informações são úteis em ambientes rurais, onde a presença de espécimes de Culicoides incomoda pela picada, geralmente irritante e dolorosa em humanos (DOWNES; WIRTH, 1981) e, que culminam com a produção de processos alérgicos em animais domésticos (MACÊDO et al., 2008).

Além disso, devido ao hábito hematófago, os Culicoides podem ser parasitados por vírus, bactérias, protozoários e vermes (SPINELLI; WIRTH, 1993). Em consequência, algumas espécies são suspeitas de serem vetores de Dirofilaria Railliet \& Henry, 1911 (BRITO et al., 2000), Onchocerca cervicalis (Railliet \& Henry, 1910) (CALVÃO-BRITO et al., 1998), Wuchereria bancrofti (Cobbold, 1877) (SHERLOCK; GUITTON, 1967) e Mansonella Faust, 1929 (SHELLEY; COSCARÓN, 2001), assim como do vírus Oropouche (NUNES et al., 2005) e de outras viroses que causam 
patologias em bovinos, equinos, caprinos e ovinos. Do ponto de vista epidemiológico, o estudo do conteúdo estomacal dos Culicoides, para a determinação de suas fontes alimentares, permite a obtenção de informações úteis para a indicação de vertebrados que potencialmente servem de reservatórios para esses parasitas. Na medida em que os vertebrados se aproximam ou coexistam no ambiente antropizado, alguns patógenos acabam afetando o ser humano, como se observa na região amazônica, onde surtos de infecção causados pelo vírus Oropouche são frequentes (TRAVASSOS DA ROSA et al., 1996). E, também nos municípios de Porto Franco e Estreito, na região sudoeste maranhense (VASCONCELOS et al., 1989; PINHEIRO et al., 1994).

Os animais domésticos são importantes fatores de atração de populações de Culicoides para os peridomicílios (SILVA; CARVALHO, 2013). Nesses ambientes, várias espécies desses dípteros foram encontradas alimentadas com sangue de vertebrados (COSTA et al., 2013). A presença de abrigos de animais domésticos, construídos de forma desordenada e a carência de condições mínimas de saneamento básico também são condições que podem facilitar a proliferação desses insetos nos peridomicílios. Sabe-se que certas espécies de Culicoides desenvolvem-se em lamaçais, esterco de animais, buracos de árvores, tecidos vegetais em decomposição (KRUGER et al., 1990; FELIPPEBAUER; STERNHEIM, 2008) que podem estar expostos nos ambientes peridomiciliares.

Com exceção de Costa et al. (2013), os estudos sobre os Culicoides no estado do Maranhão ainda estão direcionados para o conhecimento da composição das espécies (SILVA; REBÊLO, 1999; BARROS et al., 2007; SILVA; CARVALHO, 2013). As pesquisas sobre fontes alimentares vêm se concentrando em outros dípteros nematóceros, como os flebotomíneos, devido à sua importância como vetores das leishmanioses, um grupo de doenças endêmicas no estado do Maranhão (DIAS et al., 2003; OLIVEIRA-PEREIRA et al., 2008; FONTELES et al., 2009).

Diante do exposto, o objetivo do trabalho foi identificar as fontes alimentares sanguíneas dos Culicoides em povoados rurais da ilha de São Luís, e verificar o grau de relações estabelecidas entre esses insetos e os respectivos hospedeiros sanguíneos. Acredita-se que o conhecimento de potenciais hospedeiros domésticos específicos poderia servir de indicador para predizer quais as espécies de Culicoides são propensas para ocorrer em um determinado local e, assim, antecipar a elaboração e execução de estratégias apropriadas de controle entomológico e minimizar o impacto da presença destes insetos nessas áreas.

\section{Material e Métodos}

\section{Área de estudo}

As coletas foram realizadas no município de São José de Ribamar, Ilha de São Luís, Maranhão, nos povoados rurais de Santa Maria $\left(02^{\circ} 38^{\prime} 795^{\prime \prime} \mathrm{S}\right.$

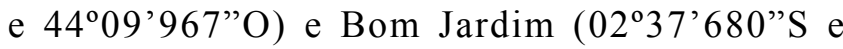
$\left.44^{\circ} 09^{\prime} 543^{\prime \prime} \mathrm{O}\right)$. Estes povoados estão distantes entre si cerca de $2 \mathrm{~km}$ e apresentando características tipicamente rurais:quintais arborizados, abrigos de animais, proximidades com brejos e riachos e com remanescentes de mata ombrófila. Essas características possibilitam aumentar a probabilidade do encontro de um maior número de espécies e maior quantidade de indivíduos disponíveis para as análises do conteúdo estomacal de Culicoides (COSTA et al., 2013). As duas localidades possuem juntas 280 casas e cerca de 1.120 habitantes e, foram implantadas praticamente na margem de uma floresta tropical chuvosa, modificada no entorno das vilas pelo corte e queima, tradicionalmente utilizados no uso do solo.

\section{Amostragem}

Foram sorteadas 10 casas que continham animais domésticos, necessários para atrair os Culicoides. Nessas casas foram realizadas as capturas dos Culicoides nos ambientes peridomésticos.

Os espécimes de Culicoides foram capturados com armadilhas luminosas tipo $\mathrm{CDC}$, das $18 \mathrm{~h}$ às 6h, sem interrupções, uma vez por mês, no período compreendido entre março de 2009 e fevereiro de 2010. No quintal de cada uma das 10 casas foi instalada uma armadilha, no ramo das árvores, a uma altura de $1,5 \mathrm{~m}$ do solo. $\mathrm{O}$ empenho de captura foi de $12 \mathrm{~h} \mathrm{x}$ 
10 armadilhas x 12 meses, resultando em $1.440 \mathrm{~h}$ de esforço amostral. Os insetos retidos nas armadilhas foram transportados para o Laboratório de Entomologia e Vetores (LEV) da Universidade Federal do Maranhão - UFMA. Em seguida, foram triados com o auxílio de estereomicroscópio Zeiss (10 a 50X) para separação de fêmeas ingurgitadas e, identificação das espécies de Culicoides, utilizando-se chaves dicotômicas propostas por Wirth e Blanton (1973), Wirth et al. (1988) e Spinelli et al. (2005). Os exemplares identificados estão acondicionados em álcool a 70\% e depositados na coleção entomológica do LEV-UFMA.

Para determinação das fontes alimentares sanguíneas, as fêmeas de Culicoides foram analisadas através do teste de reação da precipitina na Fundação Oswaldo Cruz (Fiocruz - Rio de Janeiro), onde o tubo digestório dos espécimes foi triturado em solução salina a $0,85 \%$. O macerado resultante foi mantido por $12 \mathrm{~h}$ a temperatura entre $4^{\circ}$ e $8^{\circ} \mathrm{C}$. Após esse período, foi centrifugado por 5 minutos a $1.500 \mathrm{rpm}$. O extrato sobrenadante foi exposto aos antissoros de diversos vertebrados, incluindo boi, cavalo, humano, cabra, carneiro, porco, cão, gato, tatu, gambá, roedor e ave (LOROSA et al., 1998).
Para a obtenção de dados complementares ao exame da precipitina, foi aplicado um questionário para os habitantes locais (aprovado pelo comitê de ética da UFMA, sob protocolo $n^{\circ} 5832 / 2010$ ), com perguntas referentes à presença de animais sinantrópicos e domésticos em suas propriedades. Para isso foram selecionadas para o estudo as 280 residências habitadas. Em seguida, foi realizada a aplicação dos questionários para o chefe da família ou seu representante maior de 18 anos.

\section{Resultados}

Foram capturadas 930 fêmeas ingurgitadas pertencentes a 12 espécies (Tabelas 1 e 2). As espécies de Culicoides com o maior número de indivíduos ingurgitados foram Culicoides boliviensis Spinelli \& Wirt, 1984 (18\%), seguida por C. ignacioi Forattini, 1957 (12,1\%), C. leopodoi Ortiz, $1951 \mathrm{~b}$ (12,1\%), Culicoides sp.1 (11,6\%), C. diabolicus Hoffman, 1925 (10,1\%), C. iriartei Fox, 1952 (7,2\%) e C. insignis Lutz, 1913 (6,8\%).

Entre as 930 fêmeas ingurgitadas, 888 apresentaram reações simples, 27 não reagiram com os antissoros testados (Tabela 1) e 15 tiveram reações duplas (Tabela 2).

TABELA 1: Números de reações simples do teste de precipitina em fêmeas de Culicoides ingurgitadas, no município de São José de Ribamar - MA, Brasil, nos anos de 2009 e 2010.

\begin{tabular}{l|c|c|c|c|c|c|c|c|c|c|c}
\hline \multirow{2}{*}{ Espécies de Culicoides } & \multicolumn{9}{|c|}{ Hospedeiros } & \multicolumn{3}{c}{ Total } \\
\cline { 2 - 11 } & Av & Ro & Ca & Hu & Ga & Cv & Ga & Total & NR & $\mathbf{n}^{\mathbf{0}}$ & \% \\
\hline C. boliviensis & 83 & 17 & 41 & 18 & - & - & - & 159 & 6 & 165 & 18,0 \\
C. ignacioi & 61 & 31 & 16 & - & - & - & - & 108 & 3 & 111 & 12,1 \\
C. leopodoi & 61 & 31 & 16 & - & - & - & - & 108 & 3 & 111 & 12,1 \\
C. sp.1 & 23 & 36 & 12 & 11 & - & 18 & 5 & 105 & 2 & 107 & 11,6 \\
C. diabolicus & 16 & 22 & 18 & 19 & 9 & - & 6 & 90 & 3 & 93 & 10,1 \\
C. iriartei & 27 & 16 & 13 & - & 8 & - & - & 64 & 2 & 66 & 7,2 \\
C. insignis & 25 & 9 & 8 & - & 9 & - & 8 & 59 & 4 & 63 & 6,8 \\
C. foxi & 29 & - & 9 & - & - & 18 & - & 56 & - & 56 & 6,1 \\
C. guyanensis & 8 & 19 & - & 7 & 10 & - & - & 44 & 3 & 47 & 5,1 \\
C. limai & 7 & 7 & 8 & - & - & - & 11 & 33 & - & 33 & 3,6 \\
C. paucienfuscatus & 33 & - & - & - & - & - & - & 33 & - & 33 & 3,6 \\
C. flavivenula & 11 & 6 & - & 12 & - & - & - & 29 & 1 & 30 & 3,2 \\
\hline Total & $\mathbf{3 8 4}$ & $\mathbf{1 9 4}$ & $\mathbf{1 4 1}$ & $\mathbf{6 7}$ & $\mathbf{3 6}$ & $\mathbf{3 6}$ & $\mathbf{3 0}$ & $\mathbf{8 8 8}$ & $\mathbf{2 7}$ & $\mathbf{9 1 5}$ & $\mathbf{1 0 0 , 0}$ \\
\hline \% & $\mathbf{4 1 , 9}$ & $\mathbf{2 1 , 2}$ & $\mathbf{1 5 , 4}$ & $\mathbf{7 , 3}$ & $\mathbf{3 , 9}$ & $\mathbf{3 , 9}$ & $\mathbf{3 , 2}$ & $\mathbf{9 7 , 1}$ & $\mathbf{2 , 9}$ & $\mathbf{1 0 0}$ & \\
\hline
\end{tabular}

$\mathrm{Av}=$ ave; $\mathrm{Ro}=$ roedor $\mathrm{Ca}=$ cão $\mathrm{Hu}=$ humano $\mathrm{Cv}=$ cavalo; $\mathrm{Ga}=$ galinha; $\mathrm{NR}=$ não-reagentes . 
TABELA 2: Números das reações duplas do teste da precipitina em fêmeas de Culicoides ingurgitadas no município de São José de Ribamar - MA, Brasil, nos anos de 2009 e 2010.

\begin{tabular}{|c|c|c|c|c|c|c|c|}
\hline \multirow{2}{*}{ Vertebrados } & \multicolumn{6}{|c|}{ Culicoides } & \multirow{2}{*}{ Total } \\
\hline & insignis & iriartei & guyanensis & diabolicus & ignacioi & flavivenula & \\
\hline Ave/cão & - & - & 1 & 2 & - & - & 3 \\
\hline Cão/gato & - & 2 & - & - & - & - & 2 \\
\hline Cão/gambá & - & - & - & - & - & 2 & 2 \\
\hline Ave/roedor & 2 & - & - & - & - & - & 2 \\
\hline Gato/humano & - & - & - & - & 2 & - & 2 \\
\hline Gambá/ roedor & - & - & 2 & - & - & - & 2 \\
\hline Ave/ gato & - & - & - & 1 & - & - & 1 \\
\hline Cão/cavalo & 1 & - & - & - & - & - & 1 \\
\hline Total & 3 & 2 & 3 & 3 & 2 & 2 & 15 \\
\hline
\end{tabular}

As reações simples foram confirmadas para seis tipos de animais, entre os hospedeiros domésticos (aves, cão, gato e cavalo) e sinantrópicos (roedores e gambá) e, também para o homem. As reações simples mais frequentes foram com antissoros de ave $(41,9 \%)$, roedor $(21,2 \%)$, cão $(15,4 \%)$ e humano $(7,3 \%)$ (Tabela 1$)$.

As reações duplas confirmadas representam oito combinações: cão (quatro combinações), ave e gato (três combinações), roedor e gambá (duas combinações) e humano e cavalo, com apenas uma combinação. Observou-se ainda que a reação dupla mais frequente foi ave/cão (20\%), seguida pelo cão/gato, cão/gambá, ave/roedor, gambá/roedor e gato/humano com 13,3\% cada uma (Tabela 2).

Todas as espécies de Culicoides sugaram sangue de três ou mais hospedeiros, exceto C. paucienfuscatus que sugou apenas de ave. No entanto, as espécies que utilizaram maior variedade de hospedeiros foram Culicoides sp.1 e $C$. diabolicus (seis tipos cada) e $C$. insignis (cinco).

As aves foram sugadas com mais frequência por C. leopodoi (54,9\%), C. ignacioi (54,9\%), C. foxi Ortiz, 1950 (51,7\%), C. boliviensis (50,3\%), C. iriartei (40,9\%) e $C$. insignis (39,6\%). As demais espécies de Culicoides sugaram com mais frequência outros animais, como foi o caso de Culicoides sp.1, C. flavivenula Costa Lima,1937 e C. limai Barreto, 1944 que sugaram respectivamente roedores $(33,6 \%)$, humanos $(40 \%)$ e o gambá $(33,3 \%)$, enquanto $C$. paucienfuscatus só sugou as aves.
De acordo com as informações dos moradores locais, as galinhas constituem os animais mais frequentes nos povoados (presentes no maior número de casas), tendo sido encontradas em $73,2 \%$ das casas investigadas, seguidas pelos cães $(58,6 \%)$, gatos $(41,1 \%)$ e patos (37,5\%) (Tabela 3). A Tabela 3 demonstra que as galinhas também foram os animais mais abundantes, representando 77,4\% dos animais quantificados nos peridomicílios, seguida por cães $(7,3 \%)$ e gatos $(5,5 \%)$.

Entre os animais sinantrópicos, os roedores foram citados como os mais frequentes nos peridomicílios $(21,1 \%)$, seguidos pelo gambá $(10,3 \%)$ e morcegos $(8,7 \%)$. As frequências dos demais animais estão resumidas na Tabela 4.

TABELA 3: Números de animais domésticos presentes nos peridomicílios (N1) e de casas com a presença de animais domésticos (N2) nas localidades estudadas no município de São José de Ribamar, ilha de São Luís - MA, nos anos de 2009/2010. $\mathrm{n}=$ números de casas visitadas.

\begin{tabular}{l|c|c|c|c}
\hline \multicolumn{1}{c|}{ Animais } & $\mathbf{N 1}$ & $\mathbf{\%}$ & $\mathbf{N 2} \mathbf{( n = 2 8 0 )}$ & $\mathbf{\%}$ \\
\hline Galinhas & 1.814 & 77,4 & 246 & 73,2 \\
Cães & 171 & 7,3 & 164 & 58,6 \\
Gatos & 129 & 5,5 & 115 & 41,1 \\
Patos & 120 & 5,1 & 105 & 37,5 \\
Bovinos & 40 & 1,7 & 20 & 7,1 \\
Equinos & 35 & 1,5 & 31 & 11,1 \\
Suínos & 31 & 1,3 & 18 & 6,4 \\
Araras & 2 & 0,1 & 1 & 0,4 \\
Coelho & 1 & 0,1 & 1 & 0,4 \\
\hline Total & $\mathbf{2 . 3 4 3}$ & $\mathbf{1 0 0 , 0}$ & - & - \\
\hline
\end{tabular}


TABELA 4: Número de entrevistados que citaram a presença de animais sinantrópicos nos peridomicílios das localidades estudadas no município de São José de Ribamar, ilha de São Luís - MA, nos anos de 2009/2010.

\begin{tabular}{lcc}
\hline \multicolumn{1}{c}{$\begin{array}{c}\text { Animais } \\
\text { Sinantrópicos }\end{array}$} & $\begin{array}{c}\text { Entrevistados } \\
(\mathbf{n}=\mathbf{2 8 0})\end{array}$ & $\mathbf{\%}$ \\
\hline Roedores & 59 & 21,1 \\
Gambá & 29 & 10,3 \\
Morcego & 24 & 8,7 \\
Raposa & 8 & 2,8 \\
Cobras & 7 & 2,5 \\
Guaxinim & 7 & 2,5 \\
Tatu & 5 & 1,7 \\
Quati & 5 & 1,7 \\
Camaleão & 2 & 0,7 \\
Macaco & 2 & 0,7 \\
Veado & 1 & 0,4 \\
Pássaros & 1 & 0,4 \\
Gato Maracajá & 1 & 0,4 \\
\hline
\end{tabular}

\section{Discussão}

O estudo constatou que os Culicoides encontrados apresentaram um comportamento generalista, pois sugaram tanto animais domésticos como sinantrópicos, além de o sangue humano. Apresentaram alta capacidade de picar com avidez mamíferos de porte variado e aves, corroborando as observações de Costa et al. (2013), obtidas na região dos Lençois Maranhenses. Tal comportamento é comum entre insetos nematóceros hematófagos, incluindo os flebotomíneos que foram estudados neste aspecto, também na ilha de São Luís (DIAS et al., 2003) e no interior do estado do Maranhão (OLIVEIRA-PEREIRA et al., 2008; FONTELES et al., 2009).

Apesar da maioria das espécies de Culicoides ter sugado uma variedade de vertebrados, as aves constituíram os animais mais procurados, talvez por serem os mais frequentes nas habitações e de maior densidade nos ambientes peridomésticos, conforme o depoimento dos moradores locais. Neste caso, pode-se afirmar que os Culicoides também são oportunistas, por atacar com mais avidez, o animal disponível no ambiente.
Na região dos Lençois Maranhenses, Costa et al. (2013) relataram que as aves também foram os animais mais atacados $(31,9 \%)$, seguido por cavalo e roedor com $13,8 \%$ e $12,5 \%$, respectivamente. Entretanto, no município de Raposa - MA, os percentuais de flebotomíneos ingurgitados com sangue de aves aumentou para $87,9 \%$ (DIAS et al., 2003), enquanto que em Buriticupu - MA, os animais mais sugados pelos Culicoides foram os roedores com $40 \%$ e as aves em segundo lugar com 25,8\% (OLIVEIRA-PEREIRA et al., 2008).

Neste estudo, houve o inverso, pois o roedor ocupou o segundo lugar, superando o cão. Esse resultado ocorreu devido, provavelmente, a falta de adequadas condições sanitárias na área e, remete para a necessidade de estudos mais aprofundados envolvendo esses animais como potenciais reservatórios de agentes patogênicos, visto que, os próprios moradores locais citam os ratos como os animais sinantrópicos mais frequentes nos quintais.

Considerando que o cão foi um animal frequente no ambiente doméstico e, por ser uma importante fonte de sangue para os Culicoides, há necessidade de estudá-lo como provável fonte de parasitas. Sabese que cães são hospedeiros de filárias, transmitidas por culicídeos (BRITO et al., 2000) e protozoários do gênero Leishmania Ross 1903 que têm como vetores os flebotomíneos (DIAS et al., 2003). Assim, a análise do conteúdo estomacal de Culicoides, além de fornecer importantes informações sobre os seus hábitos alimentares, também pode incriminá-los como possíveis transmissores de microorganismos patogênicos entre animais, em focos de determinadas doenças parasitárias, como filarioses e viroses (CALVÃO-BRITO et al., 1998). Entre as espécies encontradas neste estudo, $C$. insignis é incriminada como vetor primário do vírus da Língua Azul (MELLOR et al., 2000). A distribuição global desta doença se superpõe à distribuição de seu vetor primário (SILVA; CARVALHO, 2013).

Nesse sentido, a presença do homem como o quarto hospedeiro mais sugado no presente estudo, evidencia que além de estar incluído na dieta das fêmeas de Culicoides também pode participar de eventuais ciclos epidemiológicos de parasitas, devido ao hábito eclético destes ceratopogonídeos (LINLEY et al., 
1983; VASCONCELOS et al., 1989). Os resultados deste estudo indicam que os Culicoides organizam o seu alimento de acordo com a disponibilidade de vertebrados, apresentando uma característica peculiar aos hematófagos que é de sugar o maior número de hospedeiros (SPINELLI et al., 2005). Dessa forma, a variedade de animais que são criados nos ambientes peridomésticos, ao tornarem-se fonte alimentar de sangue, passam a ter grande significado na manutenção desses insetos no ambiente doméstico.

Um fato relevante foi o comportamento de $C$. paucienfuscatus, por ter sido encontrada exclusivamente com sangue de aves, lembrando que os animais utilizados como hospedeiros sanguíneos estavam disponíveis igualmente para todas as espécies de Culicoides nas habitações estudadas. Esse resultado corrobora as observações de Castellón et al. (1990) que consideram esta espécie ornitofílica. De qualquer modo, novos dados devem ser coletados em estudos sucessivos para confirmar essa suspeita, se há fidelidade desta espécie por hospedeiros representados por aves.

Para alguns Culicoides examinados não foi possível determinar a fonte de sangue ingerido. Este resultado pode ser explicado se considerarmos a pouca quantidade de sangue que esses ceratopogonídeos ingerem (GÓMEZ et al., 1998), associada com a baixa sensibilidade da precipitina na identificação das fontes alimentares dos insetos hematófagos (FORATTINI, 1973). Contudo, deve-se considerar também a variedade de anti-soros utilizados para a análise (CAMARGO-NEVES et al., 2007), que nem sempre contempla todos os animais presentes numa determinada área de estudo.

A partir dos resultados foi possível concluir que os Culicoides se alimentam do sangue de uma gama de vertebrados domésticos e sinantrópicos disponíveis no ambiente peridomésticos, estabelecendo com eles relações generalistas. Apenas C. paucienfusctaus, apresentou uma relação mais específica, tendo-se alimentado exclusivamente de aves.

\section{Referências}

APARÍCIO, A. A. S.; CASTELlÓN, G. E.; FONSECA, R. F. O. Distribuição de Culicoides (Diptera: Ceratopogonidae) na
Amazônia Legal através de técnicas de geoprocessamento. Revista Colombiana de Ciencia Animal, Sincelejo, v. 3, n. 2, p. 283-99, 2011.

BARROS, V. L. L.; MARINHO, R. M.; REBÊLO, J. M. M. Ocorrência de espécies de Culicoides Latreille (Diptera, Ceratopogonidae) na área metropolitana de São Luís, Maranhão, Brasil. Cadernos de Saúde Pública, Rio de Janeiro, v. 23, n. 11, p. 2789-2790, 2007.

BORKENT, A. World species of biting midges (Diptera: Ceratopogonidae). 2014. Disponível em $<$ http://wwx.inhs.illinois. edu/files/9913/9144/3328/CeratopogonidaeCatalog. pdf $>$. Acesso em: 26 ago. 2014.

BORKENT, A.; SPINELLI, G. R. Neotropical Ceratopogonidae (Diptera: Insecta). In: ADIS, J.; ARIAS, J. R.; RUEDA-DELGADO, G.; WNATZEN, K. M. (Ed.). Aquatic biodiversity in Latin America (ABLA). Vol. 4. Sofia-Moscow: Pensoft, 2007. p. 1-198. BRITO, A. C.; VIANA, L. S.; DUARTE, E. M.; ROCHA, E. M. M.; FONTES, G.; REGIS, L. Dirofilaria immitis infection in dogs from Maceió, Alagoas, Northeast region of Brazil. Arquivos Brasileiros de Medicina Veterinária e Zootecnia, Belo Horizonte, v. 52, n. 3, p. $210-211,2000$.

CALVÃO-BRITO, R. H. S.; RODRIGUES, M. L.; MELlO, R. P.; SILVA-JÚNIOR, V. P. Oncocercose eqüina: diagnóstico e verificação da hematofagia por simulídeos e culicóides, prováveis vetores no Rio de Janeiro, Brasil. Revista Brasileira de Zoologia, Curitiba, v. 15, n. 3, p. 583-587, 1998.

CAMARGO-NEVES, V. L. F.; RODAS, L. A. C.; GOMES, A. C. Avaliação do hábito alimentar de Lutzomyia longipalpis no estado de São Paulo. Boletim Epidemiológico Paulista, São Paulo, v. 4, n. 39, p. 4-9, 2007

CASTELlÓN, E. G.; FERREIRA, R. L. M.; SILVA, M. N. T. Culicoides (Diptera, Ceratopogonidae) na Amazônia brasileira, I. Coletas na usina hidrelétrica (UHE) de Balbina, Usina Hidrelétrica (UHE) Cachoeira Porteira e Cachoeira dos Espelhos (Rio Xingú). Acta Amazonica, Manaus, v. 20, p. 77-81, 1990.

COSTA, J. C.; LOROSA, E. S.; MORAES, J. L. P.; REBÊLO, J. M. M. Espécies de Culicoides (Diptera; Ceratopogonidae) e hospedeiros potenciais em área de ecoturismo do Parque Nacional dos Lençóis Maranhenses, Brasil. Revista Pan-Amazônica de Saúde, Belém, v. 4, n. 3, p. 11-18, 2013.

DIAS, P. O. P; LOROSA, E. S; REBÊLO, J. M. M. Fonte alimentar sanguínea e a peridomiciliação de Lutzomia longipalpis (Lutz \& Neiva) (Psycodidae, Phlebotominae). Cadernos de Saúde Pública, Rio de Janeiro, v. 19, n. 5, p. 1373-1380, 2003.

DOWNES. J. A.; WIRTH, W. W. Ceratopogonidae. In: McALPINE, J. F. (Ed.). Manual of Neartic Diptera. Quebec: Research Branch Agriculture Canadá, 1981. p. 393-421.

FELIPPE-BAUER, M. L.; OLIVEIRA, S. J. Lista dos exemplares tipos de Ceratopogonidae (Diptera: Nematocera) depositados na coleção entomológica do Instituto Oswaldo Cruz, Rio de Janeiro, Brasil. Memórias do Instituto Oswaldo Cruz, Rio de Janeiro, v. 96, n. 8, p. 1109-19, 2001.

FELIPPE-BAUER, M. L.; STERNHEIM, U. S. Culicoides paraensis (Diptera: Ceratopogo-nidae) infestations in cities of the Itapocú River Valley, Southern Brazil. Entomological News, Philadelphia, v. 119, n. 2, p. 185-192, 2008. 
FONTELES, R. S.; VASCONCELOS, G. C.; AZEVÊDO, P. C. B.; MORAES, J. L. P.; LOROSA, E. S.; LOPES, G. N. Preferência alimentar sanguínea de Lutzomyia whitmani (Diptera, Psychodidae) em área de transmissão de leishmaniose cutânea americana no estado do Maranhão, Brasil. Revista Brasileira de Medicina Tropical, Uberaba, v. 42, n. 6, p. 647-650, 2009.

FORATTINI, O. P. Subgênero Lutzomyia França, 1924. In: FORATTINI, O. P. (Ed.). Entomologia Médica: Psychodidae. Phlebotominae. Leishmanioses. Bartolenose. Vol. 4. São Paulo: Editora Edgard Blucher e Editora da Universidade de São Paulo, 1973. p. 212-228.

GOMÉZ, B.; SANCHEZ, E.; FELICIANGELI. M. D. Man-vector contact of Phlebotomine sand flies (Diptera: Psychodidae) in North-Central Venezuela as assessed by blood meal identification using DOT-ELISA. Journal of the American Mosquito Control Association, Washington, v. 14, n. 1, p. 28-32, 1998.

KRUGER, E. L.; PAPPAS, L. G.; PAPPAS, C. D. Habitat and temporal partitioning in tree hole Culicoides (Diptera: Ceratopogonidae). Journal of the American Mosquito Control Association, Washington, v. 6, n. 3, p. 390-393, 1990.

LINLEY, J. R; HOCH, A. L.; PINHEIRO, F. P. Biting midges (Diptera: Ceratopogonidae) and human health. Journal of Medical Entomology, Lanham, v. 20, n. 4, p. 347-364, 1983.

LOROSA, E. S.; ANDRADE, R. E.; SANTOS, S. M.; PEREIRA, C. A. Estudo da infecção natural e da fonte alimentar do Triatoma sordida (Stal, 1859) (Hempitera: Reduviidae) na região norte de Minas Gerais, Brasil, através da reação de precipitina. Entomologia y Vectores, Rio de Janeiro, v. 5, n. 1, p. 13-22, 1998.

MACÊDO, J. T. S. A.; RIET-CORREA, F.; DANTAS, A. F. M.; SIMÕES, S. V. D. Doenças da pele em ovinos e caprinos no semiárido brasileiro. Pesquisa Veterinária Brasileira, Seropédica, v. 28, n. 12, p. 633-642, 2008.

MEISWINKEL, R.; VENTER, G.; NEVILL, E. Vectors: Culicoides spp. In: COETZER, J.; TUSTIN, R. (Ed.). Infectious diseases of livestock. Cape Town: Oxford University Press, 2004. p. 93-136.

MELLOR, P. S.; BOORMAN, J.; BAYLIS, M. Culicoides bitings: their role as arbovirus vectors. Annual Review of Entomology, Palo Alto, v. 45, p. 307-340, 2000.

MISSAWA, N. A.; LOROSA, E. S.; DIAS, E. S. Preferência alimentar de Lutzomyia longipalpis (Lutz \& Neiva, 1912) em área de transmissão de leishmaniose visceral em Mato Grosso. Revista da Sociedade Brasileira de Medicina Tropical, Uberaba, v. 41, n. 4, p. 365-368, 2008.

NUNES, M. R. T.; MARTINS, L.C.; RODRIGUES, S. G.; CHIANG, J. O.; AZEVEDO, R. S. S.; ROSA, A. P.; VASCONCELOS, P. F. Oropouche virus isolation, Southeast Brazil. Emerging Infectious Diseases, Atlanta, v. 11, n. 10, p.1610-1613, 2005.

OLIVEIRA-PEREIRA, Y. N; MORAES, J. L. P.; LOROSA, E. S.; REBÊLO, J. M. M. Preferência alimentar sangüínea de flebotomíneos da Amazônia do Maranhão, Brasil. Cadernos de Saúde Pública, Rio de Janeiro, v. 24, n. 9, p. 2183-2186, 2008.
PINHEIRO, F. P; TRAVASSOS DA ROSA, A. P. A.; VASCONCELOS, P. F. C. Oropouche fever. In: BERAN, G. W. (Ed.). Handbook of zoonoses. 2 ed. Bocara: CRC Press, 1994. p. 214-217.

SHELLEY, A. J.; COSCARÓN, S. Simuliid blackflies (Diptera: Simuliidae) and ceratopogonid midges (Diptera: Ceratopogonidae) as vectors of Mansonella ozzardi (Nematoda: Onchocercidae) in northern Argentina. Memórias do Instituto Oswaldo Cruz, Rio de Janeiro, v. 96, n. 4, p. 451-458, 2001

SHERLOCK, I. A.; GUITTON, N. Sobre a possibilidade da transmissão da filaria Wuchereria bancrofti pelos dípteros Culicoides em Salvador, Bahia. Revista Brasileira de Malariologia e Doencas Tropicais, Rio de Janeiro, v. 19, n. 1, p. 53-61, 1967.

SILVA, F. S.; CARVALHO, L. P. C. A population study of the Culicoides biting midges (Diptera: Ceratopogonidae) in urban, rural, and forested sites in a cerrado area of Northeastern Brazil. Annals of the Entomological Society of America, Washington, v. 106, n. 4, p. 463-470, 2013.

SILVA, F. S.; REBÊLO, J. M. M. Espécies de Culicoides Latreille (Diptera: Ceratopogonidae) da ilha de São Luís, Maranhão, Brasil. Boletim do Museu Paraense Emílio Goeldi, Belém, v. 15, n. 2, p. 169-179, 1999.

SPINELLI, G. R.; RONDEROS, M. M.; DÍAZ, F.; MARINO, P. I. The bloodsucking biting midges of Argentina (Diptera: Ceratopogonidae). Memórias do Instituto Oswaldo Cruz, Rio de Janeiro, v. 100, n. 2, p. 137-150, 2005.

SPINELLI, G. R.; WIRTH, W. W. Los Ceratopogonidae de la Argentina (Insecta: Diptera). In: CASTELLANOS, Z. A. (Ed.). Fauna de agua dulce de República Argentina. Buenos Aires: Profadu (Conicet), 1993. p. 1-124.

TRAVASSOS DA ROSA, A. P. A.; RODRIGUES, S. G.; NUNES, M. R. T.; MAGALHÃES, M. T. F.; TRAVASSOS DA ROSA, J. F. S.; VASCONCELOS, P. F. C. Epidemia de febre Oropouche em Serra Pelada, município de Curionópolis, Pará, 1994. Revista da Sociedade Brasileira de Medicina Tropical, Uberaba, v. 29, n. 6, p. 537-541, 1996.

VASCONCELOS, P. F. C; TRAVASSOS DA ROSA, J. F. S.; GUEIRREIROS, S. C.; DEGALIER, N.; TRAVASSOS DA ROSA, A. P. A. Primeiro registro de epidemias causadas pelo vírus Oropouche nos estados do Maranhão e Goiás, Brasil. Revista Instituto Medicina Tropical, São Paulo, v. 31, n. 4, p. 271-279, 1989.

WIRTH, W. W.; BLANTON, F. S. A rewiew os maruins or biting midges of the genus Culicoides (Diptera: Ceratopogonidae) in the Amazon Basin. Amazoniana, Munique, v. 4, n. 4, p. 405-470, 1973.

WIRTH, W. W; DYCE, A. L.; SPINELLI, G. R. An atlas on wing photographs, with a summary of the numerical characters of the Neotropical species of Culicoides (Diptera: Ceratopogonidae). Contribution of American Entomology Institute, Gainesville, v. 25, n. 1, p. 1-72, 1988. 